\title{
Avaliação da Incontinência Urinária na Gravidez e no Pós-Parto: Estudo Observacional
}

\author{
Assessment of Urinary Incontinence in Pregnancy and \\ Postpartum: Observational Study
}

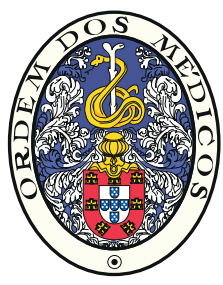

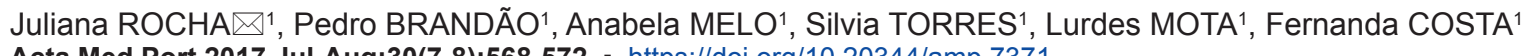

Acta Med Port 2017 Jul-Aug;30(7-8):568-572 - https://doi.org/10.20344/amp.7371

\section{RESUMO}

Introdução: A incontinência urinária pode afetar até $50 \%$ das mulheres em alguma fase das suas vidas, nomeadamente durante a gravidez. Este estudo foi desenhado com a finalidade de identificar e avaliar a prevalência e fatores de risco para incontinência urinária durante o terceiro trimestre da gravidez e três meses após o parto.

Material e Métodos: Estudo observacional e transversal. A população do estudo incluiu 268 puérperas, que tiveram parto no Centro Hospitalar Tâmega e Sousa, em 2013 e 2014. Foram avaliadas durante o período de internamento após o parto. As parturientes foram convidadas a preencher um questionário adaptado, International Consultation on Incontinence Questionnaire - Short Form, para investigação da incontinência urinária no terceiro trimestre da gravidez, para o qual deram consentimento. Três meses após o parto, foram contactadas telefonicamente e convidadas a responder às mesmas questões acerca da incontinência urinária no pós-parto.

Resultados: Das 268 mulheres entrevistadas, 31 foram excluídas do estudo, tendo em conta os critérios de inclusão e exclusão definidos. No total $(n=237), 51,89 \%$ das mulheres incluídas no estudo, relataram a ocorrência de incontinência urinária durante a gravidez. A prevalência da incontinência urinária na gravidez, segundo a paridade (primíparas versus multíparas), foi estatisticamente significativa $(p=0,006)$. No pós-parto $(n=237), 28,69 \%$ das mulheres com incontinência urinária tiveram parto vaginal e $5,91 \%$ das mulheres foram submetidas a cesariana $(p=0,001)$. Neste grupo de mulheres com incontinência urinária pós-parto $(n=82), 31,69 \%$ apresentaram incontinência urinária de novo e $68,31 \%$ das mulheres já apresentavam sintomatologia durante a gravidez $(p<0,001)$. Discussão: Este estudo demonstra a elevada prevalência da incontinência urinária na gravidez e a respetiva redução no pós-parto. Conclusão: A multiparidade e a ocorrência de incontinência urinária na gravidez surgem como possíveis fatores de risco no aparecimento da incontinência urinária.

Palavras-chave: Complicações na Gravidez; Gravidez; Incontinência Urinária; Período Pós-Parto

\section{ABSTRACT}

Introduction: The urinary incontinence can affect up to $50 \%$ of women at some stage of their lives, particularly during pregnancy and postpartum. This study was designed in order to identify and assess the prevalence and risk factors for urinary incontinence during the third trimester of pregnancy and three months postpartum.

Material and Methods: Observational and cross-sectional study. The population of the study was composed of 268 women who delivered and were admitted to the Centro Hospitalar Tâmega e Sousa in the years 2013 and 2014, and who agreed to participate in this study. Postpartum women were asked to fill out a questionnaire adapted from the International Consultation on Incontinence Questionnaire - Short Form, for urinary incontinence research in the third trimester of pregnancy. Three months after delivery, they were contacted by telephone and asked to answer the same questions about the urinary incontinence postpartum.

Results: Of the 268 women interviewed, 31 were excluded from the study, taking into account the defined inclusion and exclusion criteria. In total $(n=237), 51.89 \%$ of women included in the study, reported the occurrence of urinary incontinence during pregnancy. The prevalence of urinary incontinence in pregnancy by parity (primiparous versus multiparous) was statistically significant $(p=0.006)$. At postpartum $(n=237), 28.69 \%$ of women with urinary incontinence had vaginal delivery and $5.91 \%$ of women underwent cesarean delivery $(p=0.001)$. In these group of women with postpartum urinary incontinence $(n=82), 31.69 \%$ have had urinary incontinence only in the postpartum and $68.31 \%$ of women have had symptoms during pregnancy $(p<0.001)$.

Discussion: This study demonstrates the high prevalence of urinary incontinence in pregnancy and the respective decrease in postpartum.

Conclusion: Multiparity and occurrence of urinary incontinence in pregnancy appear as potential risk factors in the emergence of the urinary incontinence.

Keywords: Postpartum Period; Pregnancy; Pregnancy Complications; Urinary Incontinence

\section{INTRODUÇÃO}

A perda da continência urinária pode afetar até $50 \%$ das mulheres em alguma fase das suas vidas. ${ }^{1} \mathrm{O}$ estado gravídico, pelas alterações anatómicas e fisiológicas subjacentes, pode aumentar a suscetibilidade ao aparecimento de incontinência urinária (IU). Esta condição patológica é definida pela International Continence Society (ICS), como qualquer perda involuntária de urina, que pode ser classificada como de esforço ou de stress (associada a manobras de valsava e atividade física) e/ou de urgência (vontade imperiosa de urinar). ${ }^{2}$

A prevalência da IU em mulheres grávidas é variável, de 0,7 a $35 \%$, com maior impacto durante o terceiro trimestre da gestação e diminui significativamente no pós-parto..$^{2-4}$

Historicamente, a gravidez e o parto têm sido considerados fatores de risco para o aparecimento de IU no sexo feminino. A fisiopatologia subjacente permanece

1. Serviço de Ginecologia e Obstetrícia. Centro Hospitalar Tâmega e Sousa. Penafiel. Portugal.

$\triangle$ Autor correspondente: Juliana Rocha. julianasr.3@gmail.com

Recebido: 06 de janeiro de 2016 - Aceite: 04 de julho de 2017 | Copyright @ Ordem dos Médicos 2017 
Tabela 1 - Caracterização da amostra

\begin{tabular}{lc}
\hline Variável & Distribuição \\
\hline Idade (anos) & $29,25 \pm 5,66$ \\
Índice de massa corporal, prévio à gravidez $\left(\mathrm{kg} / \mathrm{m}^{2}\right)$ & $25,12 \pm 4,05$ \\
Índice de massa corporal, terceiro trimestre $\left(\mathrm{kg} / \mathrm{m}^{2}\right)$ & $31,04 \pm 4,95$ \\
Paridade & $108(45,60 \%)$ \\
Primíparas & $126(53,16 \%)$ \\
Multíparas (2 gravidezes) & $3(1,26 \%)$ \\
Multíparas ( $\geq 3$ gravidezes) & 3 \\
\hline
\end{tabular}

desconhecida, embora sejam especulados alguns fatores etiológicos, nomeadamente, o sistema hormonal, alterações no ângulo uretrovesical ou outros defeitos anatómicos e funcionais ao nível da musculatura do pavimento pélvico. ${ }^{5}$

Ainda que a IU não coloque diretamente em risco a vida da mulher, é uma condição com grande impacto psicossocial, afetando significativamente a sua qualidade de vida. No entanto, nem sempre é reconhecida, e encontra-se frequentemente subdiagnosticada e subtratada.

Com base no exposto, este estudo foi desenhado com a finalidade de identificar e avaliar a prevalência e possíveis fatores de risco para IU durante o terceiro trimestre da gravidez e três meses após o parto.

\section{MATERIAL E MÉTODOS}

Trata-se de um estudo observacional e transversal, que englobou uma amostra de 268 mulheres que tiveram o parto no Centro Hospitalar Tâmega e Sousa nos anos 2013 e 2014 e que aceitaram participar no presente estudo. Todas as mulheres englobadas assinaram previamente um consentimento informado. O protocolo do estudo foi aprovado pela Comissão de Ética do Centro Hospitalar. Os critérios de inclusão foram: a idade igual ou superior a 18 anos, com entendimento da língua portuguesa escrita e falada, sem uso atual de fármacos parassimpaticomiméticos ou simpaticolíticos e sem diabetes mellitus. Os critérios de exclusão foram: antecedentes de incontinência urinária, infeções do trato génito-urinária e de cirurgia uro-ginecológica, parto atual pré-termo, gravidez múltipla e morte fetal.

As mulheres que se encontravam internadas após o parto, foram convidadas a participar neste estudo. Efetuou-se o registo das características demográficas, peso, altura, presença de co-morbilidades e, ainda, a história obstétrica. Após explicação e assinatura do consentimento, responderam a um questionário individual e anónimo, adaptado, International Consultation on Incontinence Questionnaire Short Form (ICIQ-SF), validado para investigação da IU. ${ }^{6}$ Três meses após o parto, foram contactadas telefonicamente e convidadas a responder ao mesmo questionário [http://www.actamedicaportuguesa.com/revista/index.php/ amp/article/view/7371/5108]. Para além disso, na presença de IU, foram inquiridas sobre a orientação que procuraram, quer durante a gravidez, como no pós-parto.

A análise de dados foi efetuada utilizando o programa SPSS ${ }^{\circledR}$ versão 22.0 01/12/13 e os valores de $p<0.05$ foram considerados estatisticamente significativos. O teste $t$ Stu- dent foi utilizado para comparar grupos de mulheres com ou sem IU e variáveis quantitativas. Em relação às variáveis qualitativas, os grupos foram comparados utilizando o teste chi-square.

\section{RESULTADOS}

Das 268 mulheres entrevistadas, 31 foram excluídas do estudo, tendo em conta os critérios de inclusão. As características da amostra estão descritas na Tabela 1. No total, $51,89 \%$ das mulheres incluídas no estudo $(n=237)$, relataram a ocorrência de IU durante a gravidez; neste grupo, a IU de stress prevaleceu comparativamente à IU de urgência ou mista (82,11\% vs 17,88\%) (Fig. 1).

Foi encontrada diferença estatisticamente significativa entre a IU na gravidez e a paridade ( $p=0,006$, Fig. 2 ).

Durante a assistência pré-natal, $48,5 \%$ das mulheres relataram que não foram abordadas acerca dos sintomas de incontinência urinária.

A prevalência de IU no pós-parto foi de 34,60\% (82/237) e mais uma vez prevaleceu a IU de stress $(82,93 \%)$ versus a IU de urgência ou mista (9,76\% e 15,85\%).

Para o estudo do impacto da via de parto e de outros fatores, na ocorrência de IU no pós-parto, consideraram-se três grupos: Total da Amostra; Primíparas e Mulheres com referência de IU de novo após o parto (IU de novo) (Tabela 2).Verificou-se diferença estatisticamente significativa entre a IU e o tipo de parto quando analisado o total da amostra $(n=237 ; p=0,001)$ e especificamente no grupo de mulheres que apresentaram sintomas de novo no pós-parto ( $n=115 ; p=0,017)$. Os outros fatores analisados, nomeadamente, a realização de episiotomia, a ocorrência de laceração perineal e o peso do recém-nascido, não se

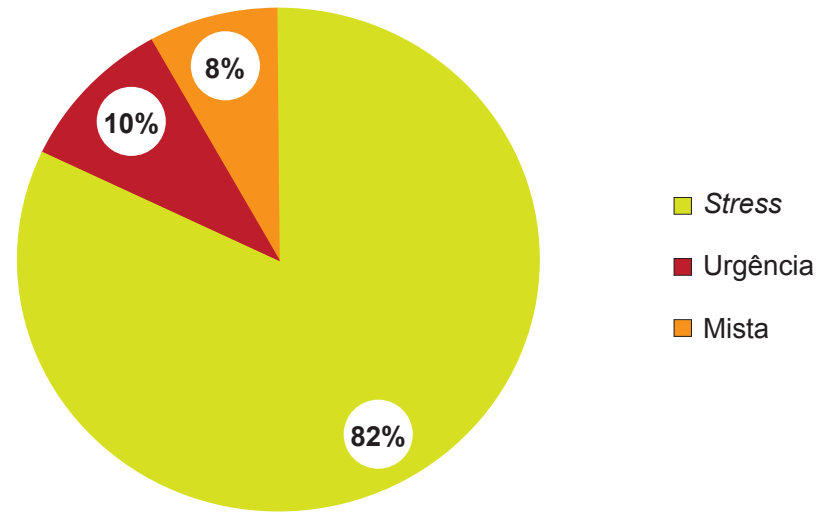

Figura 1 - Prevalência da incontinência urinária na gravidez ( $\mathrm{n}$ = 123), segundo o tipo 


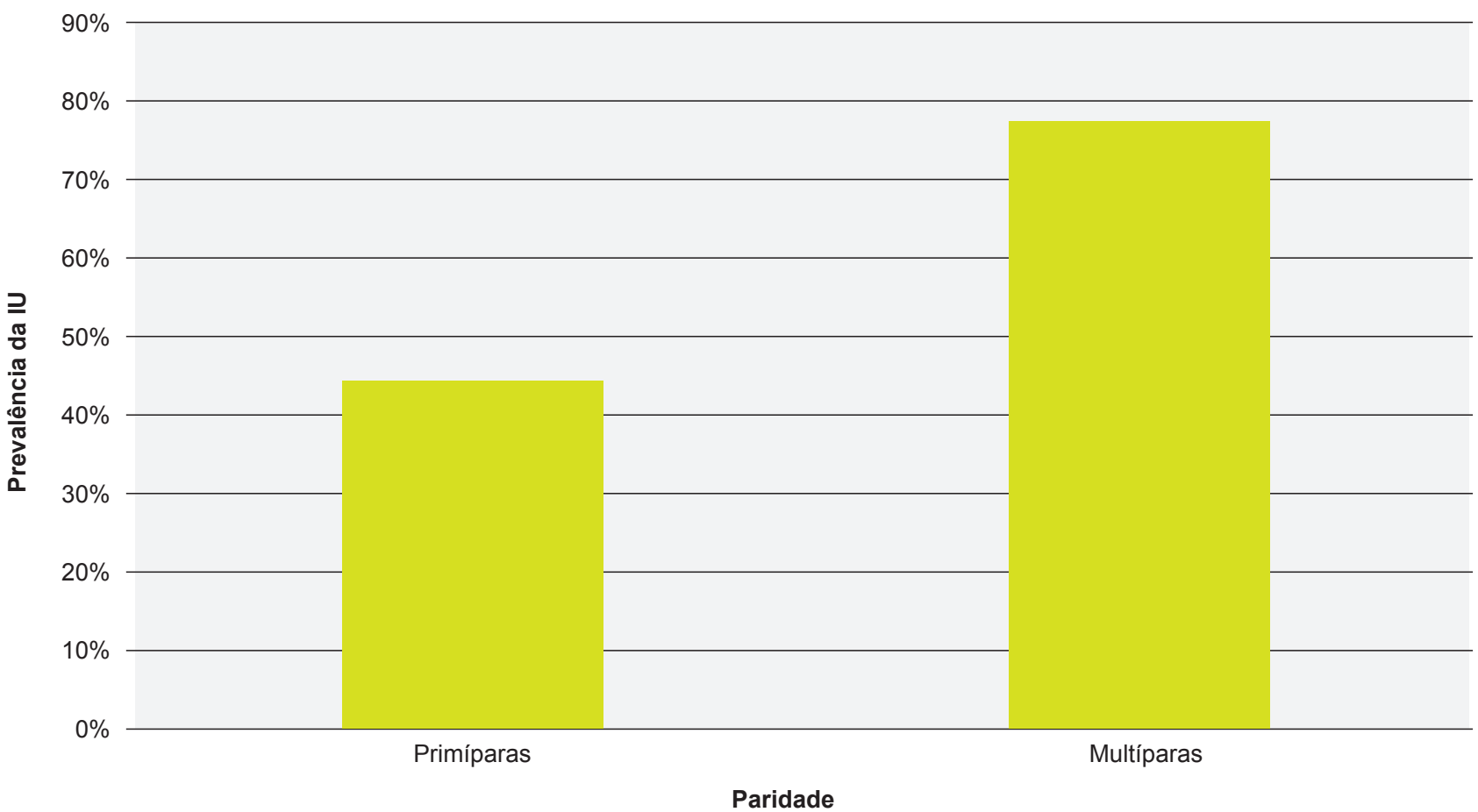

Figura 2 - Prevalência da incontinência urinária na gravidez $(\mathrm{n}=237)$, segundo a paridade $(p=0,006)$

correlacionaram com os sintomas de IU no período pós-parto nos três grupos estudados $(p>0,005)$.

No grupo de mulheres com IU pós-parto $(n=82)$, $31,69 \%$ apresentaram IU de novo e $68,31 \%$ das mulheres já apresentava sintomatologia durante a gravidez $(p<$ 0,001 ; Fig. 3). Nesta fase pós-parto, $73,41 \%$ das mulheres com IU, não procuraram aconselhamento médico.

\section{DISCUSSÃO}

O presente estudo demonstra que a prevalência da IU no terceiro trimestre da gravidez é de $51,89 \%$, compatível com os resultados de outros estudos publicados, os quais relatam taxas variáveis entre os $18,6 \%$ a $75 \% .^{7-9}$ Estas diferenças podem ser explicadas pelo tipo de metodologia utilizada para avaliar a existência de IU, variações na fase de gestação em que é estudada e possivelmente, por influência de fatores genéticos e ambientais. Para além disso, esta variabilidade permite também inferir que as alterações fisiológicas da gravidez não induzem IU em todas as mu-
Iheres. Hilde et al relatam que as mulheres nulíparas com IU, apresentam alterações na avaliação manométrica ao nível da musculatura do pavimento pélvico, sugerindo que, para além da gravidez, são múltiplos os fatores que podem diminuir a resistência do assoalho pélvico. ${ }^{10}$ Tal como está descrito na literatura, a IU de stress é o tipo mais comum na gravidez. ${ }^{11}$ Contudo, o mecanismo exato que conduz ao desenvolvimento da IU durante a gravidez permanece desconhecido. ${ }^{12}$ Têm sido implicadas alterações mecânicas e hormonais. As alterações mecânicas estão relacionadas com o aumento da pressão intra-abdominal e as respetivas repercussões no pavimento pélvico..$^{13-15}$ Por outro lado, o balanço hormonal característico da gravidez (níveis aumentados de progesterona) tem também um efeito relaxante na musculatura lisa do assoalho pélvico e está associado a uma diminuição dos níveis de colagénio. ${ }^{16,17}$

De acordo ainda com os achados do presente estudo, também vários investigadores identificaram uma associação significativa entre a multiparidade e a ocorrência de

Tabela 2 - Variáveis relacionadas com a incontinência urinária (IU) pós-parto no total da amostra, primíparas e naquelas com IU pela primeira vez manifestada após o parto (IU de novo).

\begin{tabular}{|c|c|c|c|c|c|c|c|c|c|}
\hline \multirow[t]{2}{*}{ Variáveis } & \multicolumn{3}{|c|}{$\begin{array}{l}\text { Total na amostra } \\
(\mathrm{n}=\mathbf{2 3 7})\end{array}$} & \multicolumn{3}{|c|}{$\begin{array}{l}\text { Primíparas } \\
(n=108)\end{array}$} & \multicolumn{3}{|c|}{$\begin{array}{l}\text { IU de novo } \\
(n=115)\end{array}$} \\
\hline & Sem IU & Com IU & Valor $p$ & Sem IU & Com IU & Valor $p$ & Sem IU & Com IU & Valor $p$ \\
\hline \multicolumn{10}{|l|}{ Tipo de parto } \\
\hline $\begin{array}{l}\text { Vaginal } \\
\text { Cesariana }\end{array}$ & $\begin{array}{l}41,35 \% \\
24,05 \%\end{array}$ & $\begin{array}{c}28,69 \% \\
5,91 \%\end{array}$ & 0,001 & $\begin{array}{l}49,07 \% \\
29,63 \%\end{array}$ & $\begin{array}{c}15,74 \% \\
5,55 \%\end{array}$ & 0,219 & $\begin{array}{l}42,61 \% \\
33,91 \%\end{array}$ & $\begin{array}{c}18,26 \% \\
4,35 \%\end{array}$ & 0,017 \\
\hline Episiotomia & $37,13 \%$ & $23,21 \%$ & 0,081 & $45,37 \%$ & $15,74 \%$ & 0,319 & $41,74 \%$ & $16,52 \%$ & 0,212 \\
\hline Laceração perineal & $12,66 \%$ & $6,33 \%$ & 0,149 & $17,59 \%$ & $4,62 \%$ & 0,430 & $12,17 \%$ & $4,35 \%$ & 0,461 \\
\hline $\begin{array}{l}\text { Peso do RN } \\
\quad \geq 4000 \mathrm{gr} \\
<4000 \mathrm{gr}\end{array}$ & $\begin{array}{c}4,22 \% \\
61,18 \%\end{array}$ & $\begin{array}{c}1,26 \% \\
33,33 \%\end{array}$ & 0,282 & $\begin{array}{c}2,78 \% \\
75,92 \%\end{array}$ & $\begin{array}{c}0 \% \\
21,30 \%\end{array}$ & 0,484 & $\begin{array}{c}2,61 \% \\
73,91 \%\end{array}$ & $\begin{array}{c}2,61 \% \\
20 \%\end{array}$ & 0,131 \\
\hline
\end{tabular}




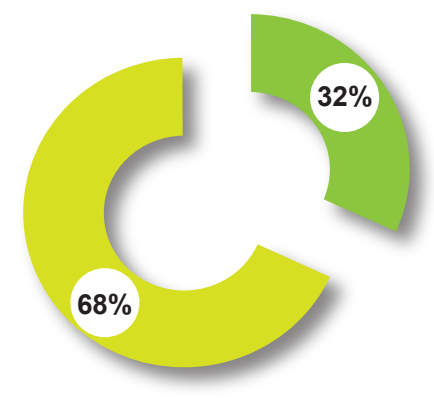

$\square$ Continente na gravidez e incontinente no pós-parto

$\square$ Incontinente na gravidez e no pós-parto

Figura 3 - Prevalência da incontinência urinária no pós-parto $(p$ $<0,001)$

IU. ${ }^{18,19}$ Segundo a literatura, estes achados suportam a hipótese de que a gravidez por si só pode exercer um efeito cumulativo negativo nos diferentes componentes da musculatura pélvica.

A IU pode permanecer durante 4 - 6 semanas pós-parto com repercussões significativas na vida da mulher. Em geral, verifica-se uma redução na prevalência da IU no pós-parto, comparativamente à gravidez (6,8\% a 42\%), sendo mais significativa se a avaliação das mulheres for realizada após um período de tempo mais alargado desde o parto. ${ }^{20-24}$ Para além disso, a prevalência de IU no pós-parto é significativamente maior quando esses sintomas ocorrem também durante a gravidez, tal como foi demonstrado pelo estudo de Martin-Martin S et al. ${ }^{25}$

Historicamente, o parto vaginal tem sido associado a um aumento do risco de IU, devido à lesão do nervo pudendo interno. ${ }^{26,27}$ Contudo, estudos mais recentes demonstraram que a cesariana não diminui o risco de IU comparativamente ao parto vaginal. ${ }^{27-29}$ Através dos resultados do presente estudo pode verificar-se uma diferença estatisticamente significativa entre o tipo de parto e a ocorrência de IU nos dois grupos de mulheres, com e sem IU na gravidez. No entanto, a multiparidade poderá constituir um fator confundidor, uma vez que no grupo de mulheres primíparas, apesar de menor dimensão, tal associação já não se verifica. A episiotomia, laceração perineal e o peso do recém-nascido, segundo estes resultados, não estão associados à ocorrência de IU. À luz dos conhecimentos atuais, a episiotomia constitui um tema de debate; embora a maioria dos estudos demonstre resultados semelhantes aos apresentados. ${ }^{30-32}$ Em relação aos restantes fatores peri-parto, alguns autores também não demonstraram qualquer associação entre a IU

\section{REFERÊNCIAS}

1. Luft J, Vriheas-Nichols AA. Identifying the risks factors for developing incontinence: can we modify individual risk? Geriatr Nurs. 1998;19:6670 .

2. Valeton CT, do Amaral VF. Evaluation of urinary incontinence in pregnancy and postpartum in Curitiba Mothers Program: a prospective study. Int Urogynecol J. 2011;22:813-8.

3. Abrams P, Cardozo L, Fall M, Griffiths D, Rosier P, Ulmsten U, et al. The standardization of terminology of lower urinary tract dysfunction:report from the Standardization Sub-committee of the International Continence Society. Neurourol Urodyn. 2002;21:167-78.

4. Gregory WT, Nygaard I. Childbirth and pelvic floor disorders. Clin Obstet Gynecol. 2004;47:394-403.

5. Menezes M, Pereira M, Hextall A. Predictors of female urinary pós-parto e a ocorrência de laceração perineal ou o peso do recém-nascido superior a 4000 gr. $^{31-34}$

Chamou-nos também a atenção o facto de que uma percentagem significativa das mulheres entrevistadas não recebeu qualquer tipo de orientação sobre a IU durante a gestação, e a maioria daquelas com IU no pós parto não procurou aconselhamento médico.

Uma das limitações do presente estudo é que todos os dados são obtidos através de uma avaliação subjetiva das mulheres (entrevista ou contacto telefónico). Para além disso, o número reduzido de mulheres em alguns dos subgrupos estudados pode não permitir alcançar resultados significativos e condicionar as conclusões definitivas relativamente aos fatores de risco periparto.

\section{CONCLUSÃO}

Este estudo demonstra a elevada prevalência da IU na gravidez e a respetiva redução no pós-parto; a multiparidade e a ocorrência de IU na gravidez, surgem como possíveis fatores de risco no aparecimento da IU. Deve ser reforçado, junto dos profissionais de saúde, a importância dos cuidados multidisciplinares e da educação para a saúde, como meios de prevenção e tratamento da disfunção do assoalho pélvico que ocorre durante a gravidez e após o parto.

\section{PROTECÇÃO DE PESSOAS E ANIMAIS}

Os autores declaram que os procedimentos seguidos estavam de acordo com os regulamentos estabelecidos pelos responsáveis da Comissão de Investigação Clínica e Ética e de acordo com a Declaração de Helsínquia da Associação Médica Mundial.

\section{CONFIDENCIALIDADE DOS DADOS}

Os autores declaram ter seguido os protocolos do seu centro de trabalho acerca da publicação de dados.

\section{CONFLITOS DE INTERESSE}

Os autores declaram não terem qualquer conflito de interesse relativamente ao presente artigo.

\section{FONTES DE FINANCIAMENTO}

Os autores declaram não ter recebido subsídios ou bolsas para a elaboração do artigo. incontinence at midlife and beyond. Maturitas. 2010;65:167-71

6. Apurologia.pt [homepage na Internet]. Orientações sobre incontinência urinária; Associação Portuguesa de Urologia. [consultado 2012 nov 30]. Disponível em http://www.apurologia.pt/guidelines/Incont-Urinaria.pdf.

7. Sangsawang B, Sangsawang N. Stress urinary incontinence in pregnant women: a review of prevalence, pathophysiology, and treatment. Int Urogynecol J. 2013;24:901-12.

8. Sharma JB, Aggarwal S, Singhal S, Kumar S, Roy KK. Prevalence of urinary incontinence and other urological problems during pregnancy: a questionnaire based study. Arch Gynecol Obstet. 2009;279:845-51.

9. Al-Mehaisen LM, Al-Kuran O, Lataifeh IM, Betawie S, Sindiyani A, Al-ttal $\mathrm{OF}$, et al. Prevalence and frequency of severity of urinary incontinence symptoms in late pregnancy: a prospective study in the north of Jordan. 
Arch Gynecol Obstet. 2009;279:499-503.

10. Hilde G, Stær-Jensen J, Ellström Engh M, Brækken IH, Bø K. Continence and pelvic floor status in nulliparous women at midterm pregnancy. Int Urogynecol J. 2012;23:1257-63.

11. Sangsawang B. Risk factors for the development of stress urinary incontinence during pregnancy in primigravidae: a review of the literature. Eur J Obstet Gynecol Reprod Biol. 2014;178;27-34.

12. Viktrup $L$. The risk of urinary tract symptom five years after the first delivery. Neurourol Urodyn. 2002;21:2-29.

13. Wijma J, Weis Potters AE, de Wolf BT, Tinga DJ, Aarnoudse JG. Anatomical and functional changes in the lower urinary tract during pregnancy. Br J Obstet Gynaecol. 2001;108:726-32.

14. Jundt K, Scheer I, Schiessl B, Karl K, Friese K, Peschers UM. Incontinence, bladder neck mobility, and sphincter ruptures in primiparous women. Eur J Med Res. 2010;15:246-52.

15. Chaliha C, Bland JM, Monga A, Stanton SL, Sultan AH. Pregnancy and derivery: a urodynamic viewpoint. $\mathrm{Br} \mathrm{J}$ Obstet Gynaecol. 2000;107:1354-9.

16. Swift SE, Ostergard DR. Effects of progesterone on the urinary tract. Int Urogynecol J. 1993;4:232-6.

17. Keane DP, Sims TJ, Abrams P, Bailey AJ. Analysis of collagen status in premenopausal nulliparous women with genuine stress incontinence. $\mathrm{Br}$ J Obstet Gynaecol. 1997;104:994-8.

18. Wesnes SL, Rortveit G, Bø K, Hunskaar S. Urinary incontinence during pregnancy. Obstet Gynecol. 2007;109:922-8.

19. Adaji SE, Shittu OS, Bature SB, Nasir S, Olatunji O. Suffering in silence: pregnant women's experience of urinary incontinence in Zaria, Nigeria. Eur J Obstet Gynecol Reprod Biol. 2010;150:19-23.

20. Wilson PD, Herbison RM, Herbison GP. Obstetric practice and the prevalence of urinary incontinence three months after delivery. $\mathrm{Br} \mathrm{J}$ Obstet Gynaecol. 1996;103:154-61.

21. Mørkved S, Bø K. Prevalence of urinary incontinence during pregnancy and postpartum. Int Urogynecol J. 1999;10:304-98.

22. Tanawattanacharoen S, Thongtawee S. Prevalence of urinary incontinence during the late third trimester and three months postpartum period in King Chulalongkorn Memorial Hospital. J Med Assoc Thai. 2013;96:144-9.

23. Zhu L, Li L, Lang JH, Xu T. Prevalence and risk factors for peri- and postpartum urinary incontinence in primiparous women in China: a prospective longitudinal study. Int Urogynecol J. 2012;23:563-72.

24. Thom DH, Rortveit G. Prevalence of postpartum urinary incontinence: a systematic review. Acta Obstet Gynecol Scand. 2010;89:1511-22.

25. Martin-Martin S, Pascual-Fernandez A, Alvarez-Colomo C, CalvoGonzalez R, Muñoz-Moreno M, Cortiñas-Gonzalez JR. Urinary incontinence during pregnancy and postpartum. Associated risk factors and influence of pelvic floor exercises. Arch Esp Urol. 2014;67:323-30.

26. Hantoushzadeh S, Javadian P, Shariat M, Salmanian B, Ghazizadeh S, Aghssa M. Stress urinary incontinence: pre-pregnancy history and affects of mode of delivery on its postpartum persistency. Int Urogynecol J. 2011;22:651-5

27. McKinnie V, Swift SE, Wang W, Woodman P, O'Boyle A, Kahn M, et al. The effect of pregnancy and mode of delivery on the prevalence of urinary and fecal incontinence. Am J Obstet Gynecol. 2005;193:512-7.

28. Rortveit G, Daltveit AK, Hannestad YS, Hunskaar S. Vaginal delivery parameters and urinary incontinence: the Norwegian EPINCONT study. Am J Obstet Gynecol. 2003;189:1268-74.

29. MacLennan AH, Taylor AW, Wilson DH, Wilson D. The prevalence of pelvic floor disorders and their relationship to gender, age, parity and mode of delivery. BJOG. 2000;107:1460-70.

30. van Brummen $H J$, Bruinse $H W$, van de Pol G, Heintz $A P$, van der Vaart $\mathrm{CH}$. Bothersome lower urinary tract symtoms 1 year after first delivery: prevalence and the effect of childbirth. BJU Int. 2006;98:89-95.

31. Torkestani F, Zafarghandi N, Davati S, Hadavand SH, Garshasbi M Case-controlled study of the relationship between delivery method and incidence of post-partum urinary incontinence. J Int Med Res. 2009;37:214-9.

32. De Lancey JO, Miller JM, Kearney R, Howard D, Reddy P, Umek W, et al. Vaginal birth and de novo stress incontinence: relative contributions of urethral dysfunction and mobility. Obstet Gynecol. 2007;110:354-62.

33. Ekström A, Altman D, Wiklund I, Larsson C, Andolf E. Planned cesarean section versus planned vaginal delivery: comparision of lower urinary tract symptoms. Int Urogynecol J Pelvic Floor Dysfunct. 2008;19:45965.

34. Boyles SA, Flood C, Schulz JA, MacDonald D, Esau D, Jones S, et al. Prevalence and risk factos for urinary and fecal incontinence four months after vaginal delivery. J Obstet Gynaecol Can. 2009;31:36-41. 